\title{
Resonant Oscillations of a Granular Cluster
}

\author{
EVGENIY KHAIN \\ Department of Physics, Oakland University, Rochester, Michigan 48309 \\ This article was submitted as an invited paper resulting from the "Understanding Complex Systems" \\ conference held at the University of Illinois-Urbana Champaign, May 2006
}

Received November 15, 2006; revised July 25, 2007; accepted July 27, 2007

\begin{abstract}
Driven granular media is a fascinating example of intrinsically non-equilibrium system. I consider an ensemble of inelastically colliding hard disks in a channel driven by two opposite thermal walls with different temperatures. When the inelasticity of collisions is large enough, a dense cluster, located closer to the colder wall, is formed. The steady state profiles are computed from the equations of granular hydrodynamics, and a good agreement with the results of molecular dynamics simulations is found. When the temperature of one of the thermal walls is varied periodically, a dense cluster develops oscillations. The oscillations amplitude reaches its maximum in a resonance, when the frequency of driving is roughly equal to the hydrodynamic frequency of oscillatory acoustic modes in the system. (c) 2008 Wiley Periodicals, Inc. Complexity 00: 00-00, 2008
\end{abstract}

Key Words: driven granular gas; molecular dynamics simulations; granular hydrodynamics; resonance; fluctuations

\section{INTRODUCTION}

$\mathrm{G}$ ranular materials are ubiquitous in nature and of great importance in industry. In the last decade granular matter (matter composed of macroscopic particles interacting dissipatively) attracted significant attention of physicists, as it presents a fascinating example of intrinsically non-equilibrium systems [1, 2]. Fluidized granular media exhibit a variety of pattern-formation phenomena [3]. In this work I focus on driven granular systems. In these systems steady states are achievable, when the external energy input into the system is balanced by inelastic heat losses in the bulk. One of the simplest settings is driving the ensemble of inelastically colliding hard

Corresponding author: Evgeniy Khain (e-mail: khain@ oakland.edu) disks by (one or) two opposite thermal walls [4-6]. To describe the system, I employ Navier-Stockes granular hydrodynamics [7], which can be derived systematically from the kinetic theory for dilute and moderately dense systems $[8,9]$. Then, I tested the hydrodynamic predictions in event-driven molecular dynamics (MD) simulations [10].

When the inelasticity of collisions between particles is significantly large, a dense static cluster of particles is formed far from the thermal walls [11]. What happens when the temperature of one of the thermal walls is varied periodically with time? It would be natural to assume that if the driving frequency is too high (so that inverse driving frequency is smaller than the characteristic transient time for cluster formation), the cluster will remain at rest. In this work, I show that this is not always the case. When the driving frequency equals to some intrinsic frequency 
of the system, a resonance occurs, and the cluster develops oscillations.

The article is organized as follows. In the next section I describe the model, solve the 1D steady state problem with constant thermal walls' temperatures, and compare the hydrodynamic predictions with the results of eventdriven MD simulations. Then I consider the problem of periodically varying thermal wall temperature and analyze resonant oscillations. The last section includes a brief discussion and a summary.

\section{THE MODEL, GOVERNING EQUATIONS, AND DESCRIPTION OF A STATIC GRANULAR CLUSTER}

Consider a two-dimensional system of inelastically colliding hard disks with unit mass and diameter $d$. There is no gravity in the system. In each collision between two particles, momentum is conserved, but some energy is lost. In the simplest model, the energy loss is characterized by the only parameter that is the coefficient of normal restitution $r$. Elastic collisions correspond to $r=1$; in the following we will assume the limit of nearly elastic collisions, $1-r<<$ 1. The two walls located at $x=0$ and $x=L$ are thermal: $T(x=0)=T_{0}, T(x=L)=T_{1}$. Upon collision with a thermal wall, the normal component of the particle velocity is taken from a Maxwell distribution with the corresponding temperature, while the tangential component of the velocity remains unchanged. The two remaining walls, located at $y$ $=0$ and $y=H$, are elastic. I start with the initially uniform spatial distribution of the particles; the initial velocity distribution is Maxwell's with a unit temperature.

Consider a static steady state. In this case, the pressure is constant. Consider first that the two opposite thermal walls have the same temperature. The granular temperature decreases, as one moves away from the thermal walls toward the middle of the system due to inelastic particle collisions, so the density increases with this distance in order to maintain constant pressure. From symmetry considerations, the density reaches its maximum in the middle of the system. If the inelasticity of particle collisions is sufficiently large, a dense granular cluster in the middle is formed. If the two thermal walls have different temperatures the cluster shifts toward the colder wall. The density and temperature profiles can be found from the steady state equations of granular hydrodynamics $[9,11]$ :

$$
\begin{gathered}
\frac{d}{d x}[n T(1+2 G)]=0, \\
\frac{d}{d x}\left(T^{1 / 2} F \frac{d T}{d x}\right)=R n G T^{3 / 2},
\end{gathered}
$$

where

$$
G=\frac{\pi n}{2 \sqrt{3}} \frac{1-\frac{7 \pi n}{32 \sqrt{3}}}{\left(1-\frac{\pi n}{2 \sqrt{3}}\right)^{2}}, \quad F=n G\left[1+\frac{9 \pi}{16}\left(1+\frac{2}{3 G}\right)^{2}\right],
$$

\section{FIGURE 1}

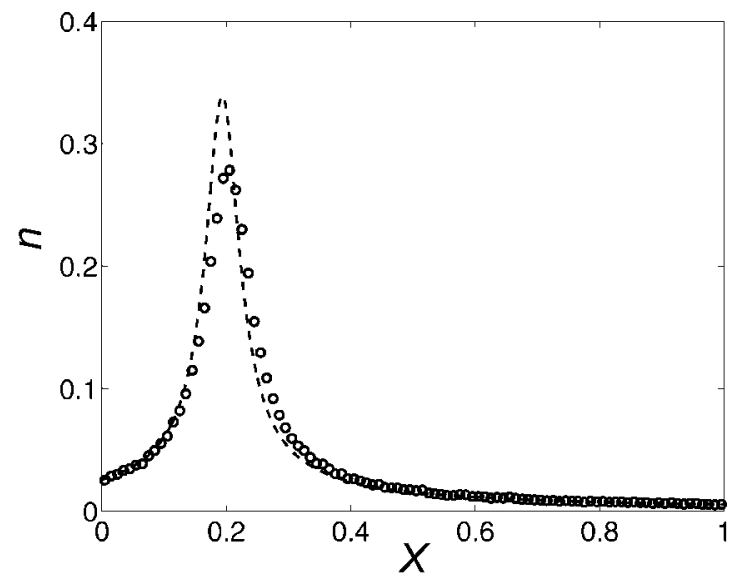

An example of a density profile, calculated both from hydrodynamic eqs. (1) and (2), dashed line, and from event-driven MD simulations, circles. The system dimensions are $L=865.57$ and $H=87.88$, the number of particles is $N=3940$, and the restitution coefficient is $r=0.99$. This corresponds to the following hydrodynamic parameters: $R=30,000, f=0.0449$, and $\delta=5$.

the distance is measured in units of $L$, the density $n(x)$ in units of the density of close packing $n_{c}=2 /\left(\sqrt{3} d^{2}\right)$, the temperature $T(x)$ in units of $T_{0}$, and $R=4(1-r) L^{2} / d^{2}$. In addition, there are two boundary conditions for the temperature $T(x=0)=1$ and $T(x=1)=\delta$, where $\delta$ is the ratio between temperatures of two opposite thermal walls $\delta=$ $T_{1} / T_{0}$. Finally, the total number of particles is conserved: $\int_{0}^{1} n(x) d x=f$, where $f=N /\left(L H n_{c}\right)$ is the average area fraction occupied by the grains.

Figure 1 shows an example of a dense cluster formed away from the thermal walls, as obtained from the numerical solution of Eqs. (1) and (2) (the solid line). To test these predictions, I performed event-driven MD simulations. After a short transient, the system reached the steady state; this was verified by following the $x$-component of center of mass of the system, $X_{\mathrm{cm}}$, see Figure 2. A density profile, calculated from MD simulations at the steady state, is also shown in Figure 1 (the circles). A good agreement between the hydrodynamic theory and MD simulations can be seen.

\section{RESONANCE CLUSTER OSCILLATIONS}

The position of the center of mass computed from the steady state hydrodynamics does not depend on time by definition; see Figure 2, the dashed line. However, the results of MD simulations show small amplitude time dependence of $X_{\mathrm{cm}}$. These small-amplitude noisy oscillations 


\section{FIGURE 2}

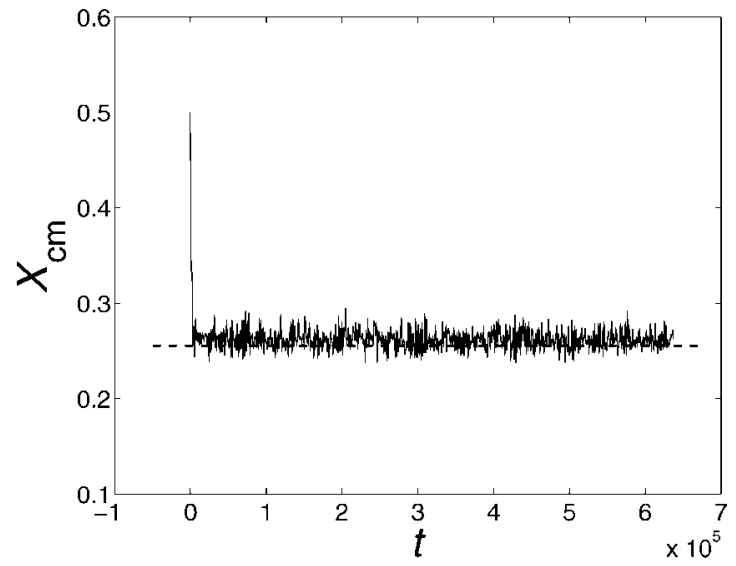

$X$-component of the center of mass of the system versus time. After a short transient, the system reaches a steady state (see Figure 1 for the density profile). The results of MD simulations are shown by the solid line; the dashed line corresponds to hydrodynamic theory. The parameters are the same as in Figure 1.

can also be seen from the corresponding power spectrum, which has a peak at some frequency $\omega_{1}$, see Figure 3. What is the origin of these oscillations? To answer this question, I linearized the full hydrodynamic equations

\section{FIRURE 3}

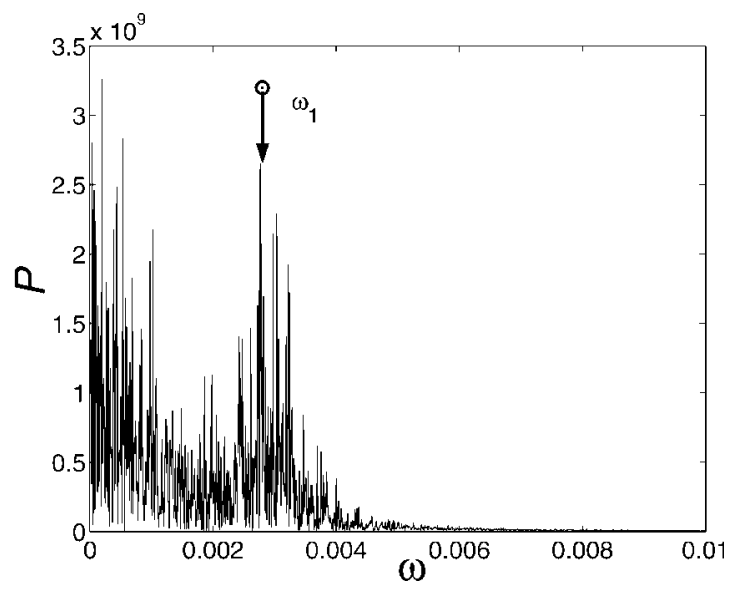

Power spectrum of $x$-component of the center of mass oscillations, as obtained from MD simulations. The characteristic hydrodynamic frequency of acoustic modes, $\omega_{2}=0.0022$, is fairly close to $\omega_{1} \cong$ 0.0028 , see text. The parameters are the same as in Figure 1. around the static steady state, similarly to what was done in [11]. There are three hydrodynamic modes in the system: two of them are oscillatory acoustic modes, and the third one is a non-oscillatory entropy mode; all three modes have negative real parts (decaying modes). The frequency of acoustic modes $\omega_{2}$ turned out to be fairly close to $\omega_{1}$, see Figure 3 . This can be explained as follows. There is the whole spectrum of noisy fluctuations in the system due to the discrete nature of particles. Amplified are those fluctuations, whose frequency approximately equals to the natural frequencies in the system, in our case, to the frequency of acoustic modes.

To explore this effect further, let us vary the temperature of one of the thermal walls periodically with time:

$$
T(x=1)=\delta+a \cos \left(\omega_{3} t\right)
$$

The resulting oscillations of $X_{\mathrm{cm}}$ become maximal when the frequency of external driving, $\omega_{3}$, equals to $\omega_{1}$. For the higher driving frequency $\omega_{3}$, the oscillations amplitude decreases significantly, as shown in Figure 4 (see also Figure 5). If the driving frequency is too low, there is a (quasi) steady state, and the cluster adapts its position to the current momentary wall temperatures. However, even in this case (for lower driving frequencies $\omega_{3}<\omega_{1}$ ) the amplitude of oscillations is smaller than in the resonance, see Figure 5. This suggests that the cluster oscillates due to a resonance, which occurs when $\omega_{3} \cong \omega_{1}$.

\section{FIGURE 4}

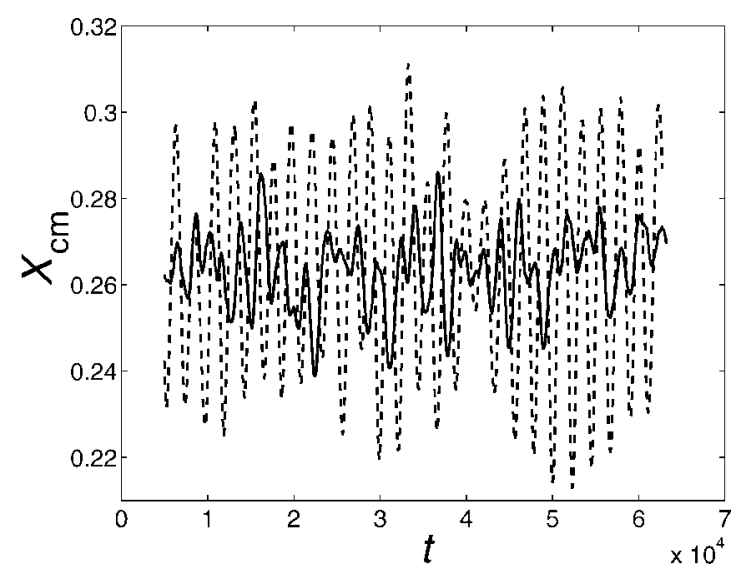

$X$-component of the center of mass oscillations both in case of resonance $\omega_{3} \cong \omega_{1}=0.0028$ (dashed line) and for a higher driving frequency $\omega_{3}=0.006$ (solid line). The temperature of the thermal wall, located at $x=1$, is given by Eq. (3) with $a=1$. The other parameters are the same as in Figure 1. 


\section{FIGURE 5}

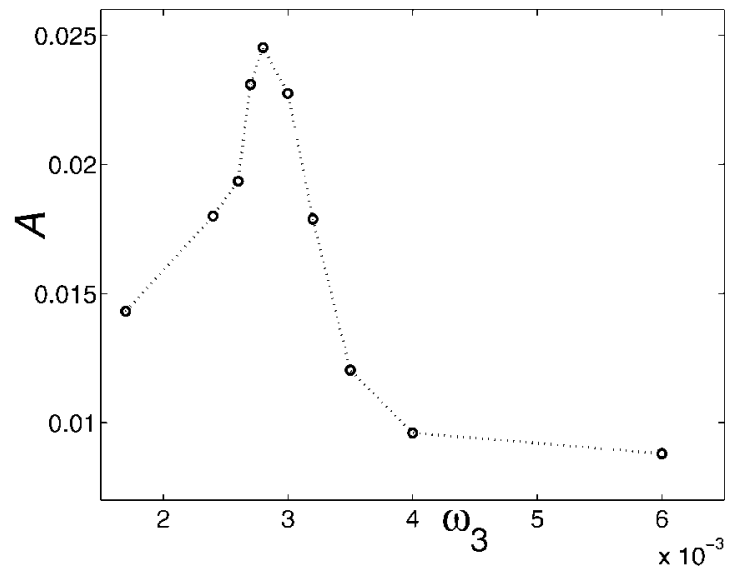

The amplitude of the center of mass oscillations as a function of driving frequency. The temperature of the thermal wall, located at $x=1$, is given by Eq. (3) with $a=1$. The other parameters are the same as in Figure 1.

\section{SUMMARY AND DISCUSSION}

In this work I considered a granular gas driven by two opposite thermal walls. For significantly high inelasticity of collisions, a dense cluster of particles is formed away from the walls. The density profile was described by the equations of granular hydrodynamics, and a good agreement with the results of event driven MD simulations was obtained. However, MD simulations showed small-amplitude noisy oscillations of the center of mass of the system. These oscillations are related to fluctuations, intrinsically present in the system due to the discrete nature of particles. The role of fluctuations in granular dynamics has received much recent attention [12-16]; fluctuations should be taken into account to quantitatively describe the behavior of granular media.

To study this effect further, the temperature of one of the thermal walls was varied periodically with time. In this case, the cluster may or may not develop oscillations depending on the driving frequency. I showed that the cluster oscilla- tions become significant when a resonant occurs: the driving frequency equals the same characteristic frequency of the system. This frequency is fairly close to the hydrodynamic frequency of the acoustic modes in the system.

Consider again the case of constant wall temperatures. Small amplitude center of mass oscillations in the system can be explained as follows. There is a wide spectrum of noisy fluctuations in the system because of the discrete nature of particles. However, amplified are those fluctuations, whose frequency approximately equals to the natural frequencies in the system, in our case, to the frequency of acoustic modes. I would like to emphasize, that in the regime of parameters, considered in the paper, the hydrodynamic acoustic modes decay, in contrast to what was observed in [11] where these modes had positive real part, leading to hydrodynamic instability.

Both granular hydrodynamics and MD simulations show that this simple clustering state can exhibit spontaneous symmetry-breaking instability. This instability leads to phase separation: coexistence of dense and dilute regions of the granulate in the lateral direction perpendicular to the (one or two) driving walls [16-23]. In this work, I considered the region of parameters where the laterally uniform stripe state is stable.

In experiments, granular medium is often fluidized by a rapidly vibrating wall. To what extent a thermal wall can model the experimental driving? Qualitatively, a thermal wall is assumed a good approximation when the vibration amplitude of the driving wall is small (much smaller than the mean free path of the particles near the wall) and the frequency of vibration is high (much higher than the particle collision rate near the wall). It is not clear how to formulate a general hydrodynamic boundary condition, which would take into account a vibrating wall. In some important cases, however, it can be done; it was shown that a constant heat flux boundary condition mimics better the vibrating wall [24-27]. Importantly, the existence of the clustering state does not depend on the exact form of the boundary conditions.

\section{ACKNOWLEDGMENTS}

I would like to thank the Michigan Center for Theoretical Physics, where this work was initiated, for support.

\section{REFERENCES}

1. Poschel, T.; Luding, S., Eds. Granular Gases; Springer: Berlin, 2001.

2. Poschel, T.; Brilliantov, N., Eds. Granular Gas Dynamics; Springer: Berlin, 2003.

3. Aranson, I.S.; Tsimring, L.S. Patterns and collective behavior in granular media: Theoretical concepts. Rev Mod Phys 2006, 78, 641-692.

4. Grossman, E.L.; Zhou, T.; Ben-Naim, E. Towards granular hydrodynamics in two dimensions. Phys Rev E 1997, 55, 42004206.

5. Esipov, S.E.; Poschel, T. The granular phase diagram. J Stat Phys 1997, 86, 1385-1395.

6. Tobochnik, J. Granular collapse as a percolation transition. Phys Rev E 1999, 60, 7137-7142. 
7. Goldhirsch, I. Rapid granular flows. Annu Rev Fluid Mech 2003, 35, 267-293.

8. Haff, P.K. Grain flow as a fluid-mechanical phenomenon. J Fluid Mech 1983, 134, 401-430.

9. Jenkins, J.T.; Richman, M.W. Kinetic theory for plane flows of a dense gas of identical rough, inelastic, circular disks. Phys Fluids 1985, 28, 3485-3494.

10. Rapaport, D.C. The Art of Molecular Dynamics Simulation; Cambridge University Press: Cambridge, 1995.

11. Khain, E.; Meerson, B. Oscillatory instability in a driven granular gas. Europhys Lett 2004, 65, 193-199.

12. Brey, J.J.; Garcia de Soria, M.I.; Maynar, P.; Ruiz-Montero, M.J. Scaling and universality of critical fluctuations in granular gases. Phys Rev Lett 2005, 94, 098001.

13. Puglisi, A.; Visco, P.; Barrat, A.; Trizac, E.; van Wijland, F. Fluctuations of internal energy flow in a vibrated granular gas. Phys Rev Lett 2005, 95, 110202.

14. Brey, J.J.; Dominguez, A.; Garcia de Soria, M.I.; Maynar, P. Mesoscopic theory of critical fluctuations in isolated granular gases. Phys Rev Lett 2006, 96, 158002.

15. Visco, P.; Puglisi, A.; Barrat, A.; van Wijland, F.; Trizac, E. Energy fluctuations in vibrated and driven granular gases. Europ Phys J B 2006, 51, 377-387.

16. Meerson, B.; Poschel, T.; Sasorov, P.V.; Schwager, T. Giant fluctuations at a granular phase separation threshold. Phys Rev E 2004, 69, 021302.

17. Livne, E.; Meerson, B.; Sasorov, P.V. Symmetry-breaking instability and strongly peaked periodic clustering states in a driven granular gas. Phys Rev E 2002, 65, 021302.

18. Brey, J.J.; Ruiz-Montero, M.J.; Moreno, F; Garcia-Rojo, R. Transversal inhomogeneities in dilute vibrofluidized granular fluids. Phys Rev E 2002, 65, 061302.

19. Khain, E.; Meerson, B. Symmetry-breaking instability in a prototypical driven granular gas. Phys Rev E 2002, 66, 021306.

20. Argentina, M.; Clerc, M.G.; Soto, R. Van der Waals-like transition in fluidized granular matter. Phys Rev Lett $2002,89,044301$.

21. Livne, E.; Meerson, B.; Sasorov, P.V. Symmetry breaking and coarsening of clusters in a prototypical driven granular gas. Phys Rev E 2002, 66, 050301(R).

22. Khain, E.; Meerson, B.; Sasorov, P.V. Phase diagram of van der Waals-like phase separation in a driven granular gas. Phys Rev E 2004, 70, 051310.

23. Cartes, C.; Clerc, M.G.; Soto, R. Van der Waals normal form for a one-dimensional hydrodynamic model. Phys Rev E 2004, 70, 031302.

24. Kumaran, V. Temperature of a granular material "fluidized" by external vibrations. Phys Rev E 1998, 57, 5660-5664.

25. Brey, J.J.; Ruiz-Montero, M.J.; Moreno, F. Boundary conditions and normal state for a vibrated granular fluid. Phys Rev E 2000, 62, 5339-5346.

26. Soto, R. Granular systems on a vibrating wall: The kinetic boundary condition. Phys Rev E 2004, 69, 061305.

27. Soto, R.; Mansour, M.M. Hydrodynamic boundary condition in vibrofluidized granular systems. Physica A 2006, 369, 301308. 